\title{
Voruntersuchungen zur berührungslosen Gestenerkennung mittels kapazitiver Sensorik
}

\author{
Leonhard Haslinger ${ }^{1}$, Bernhard G. Zagar ${ }^{1}$ \\ ${ }^{1}$ Johannes Kepler Universität Linz \\ Institut für Elektrische Messtechnik \\ Altenbergerstraße 69 \\ 4040 Linz, Österreich
}

\section{Zusammenfassung}

Dieser Beitrag beschäftigt sich mit Voruntersuchungen zur berührungslosen Gestenerkennung mittels kapazitiver Sensorik. Aufgrund der geringen Kapazitäten und der raschen zeitlichen Kapazitätsänderungen die durch Fingergesten verursacht werden, stellt deren Messung und Online-Auswertung eine große Herausforderung dar. Für eine zukünftige Positionsbestimmung zur Erkennung solcher Gesten wird daher ein physikalisches Kapazitätsmodell hergeleitet. Dieses Modell soll lediglich unter vereinfachten Bedingungen eine gute Annäherung an die Realität darstellen. Hierfür wird zunächst unter Berücksichtigung von Symmetrieeigenschaften die 3D Geometrie auf ein 2D Problem vereinfacht. Darüber hinaus wird die 2D Konfiguration für eine mathematische Beschreibung des Kapazitätsverlaufs in weitere Teilprobleme gegliedert. Anschließend werden die Teillösungen des 2D Problems zu einem Kapazitätsmodell für drei Raumdimensionen zusammengefasst. Anhand von Messungen wird die Qualität des 3D Kapazitätsmodells untersucht.

Keywords: Modellierung, kapazitive Näherungssensoren, Kapazitätsverlauf, 3D Interaktion, berührungslose Positionsbestimmung.

\section{Einleitung}

Ein Großteil der wissenschaftlichen Publikationen, die die berührungslose Erkennung von menschlichen Gesten mittels kapazitiver Sensorik behandeln, beruhen auf Machine Learning Ansätzen [1,2]. Zur Implementierung solcher Algorithmen genügen oft empirische Näherungen für den Kapazitätsverlauf [3] und ein Satz von Trainingsdaten.

Im Gegensatz dazu erfolgt im vorliegenden Beitrag, zum besseren Verständnis der physikalischen Zusammenhänge, eine mathematische Herleitung eines Kapazitätsmodells. Aufgrund der geringen Kapazitätswerte und der raschen zeitlichen Kapazitätsänderungen die durch Fingergesten verursacht werden, stellt deren Messung und Online-Auswertung eine große Herausforderung dar, da Störeffekte hier erheblichen Einfluss haben. Zukünftig soll mit einem Kapazitätsmodell zunächst auf die Position und in weiterer Folge durch eine Sequenz von Positionen auf eine mögliche Geste des Fingers geschlossen werden. Darauf aufbauend ist geplant aus der Bildverarbeitung bekannte Deskriptoren wie z.B. Fourier Deskriptoren oder Shape Numbers [4] für die Gestenerkennung zu verwenden. Hierbei soll das Kapazitätsmodell unter vereinfachten Bedingungen eine gute Annäherung an die Realität darstellen. Zum einen ist für die Gestenerkennung eine moderate Ortsauflösung ausreichend, sofern die Zeitauflösung der Messung hoch genug ist um geschmeidige Bewegungen verfolgen zu können. Zum anderen sind die Varietäten von Fingern verschiedener Personen sowie die unzähligen Möglichkeiten an Umgebungseinflüssen und Störeffekten nicht mit vertretbarem Aufwand exakt modellierbar. Außerdem kann bei der Gestenerkennung über gezielte Feedbackmaßnahmen selbst bei unzureichend erkannter Geste der Benutzer sein Verhalten unterstützend anpassen [5].

Abb. 1 zeigt die zugrunde liegende Messanordnung zu der in dieser Arbeit betrachteten Problemstellung bestehend aus dem Messobjekt mit der Fläche $a_{m} \times a_{m}$ und der Höhe $h_{m}$, der Sensorelektrode mit der Fläche $a_{s} \times a_{s}$ und der Schirmelektrode. Der menschliche Finger wird durch einen quadratischen Metallquader abstrahiert. Dessen Position wird über den Mittelpunkt $\left[x_{m}, y_{m}, z_{m}\right]^{\mathrm{T}}$ der Quadergrundfläche referenziert. Dabei wird die von der Position des Messobjekts abhängige Kapazität $C_{3 D}$ zwischen Messobjekt und Sensorelektrode untersucht. Für das umgebende Luftvolumen wird die Permittivität $\varepsilon_{0}$ des Vakuums verwendet. Auf der Sensorelektrode liegt das Messsignal an, mit dem die Kapazität gegen Erdpotential gemessen wird. Das Messobjekt ist im 
Messaufbau mit Erdpotential verbunden. Im Allgemeinen beträgt die Kapazität des menschlichen Körpers gegen Erdpotential ca. 150pF [6]. Da dieser Wert wesentlich größer ist als der zu erwartende maximale Messwert ${ }^{1}$ für $C_{3 D}$, ist eine leitende Verbindung des Quaders zum Erdpotential gerechtfertigt. Zum Schutz vor Störungen wird die Schirmelektrode über einen Verstärker auf gleichem Potential wie die Sensorelektrode gehalten. Dadurch wird die Wirkung parasitärer Kapazitäten eingeschränkt.

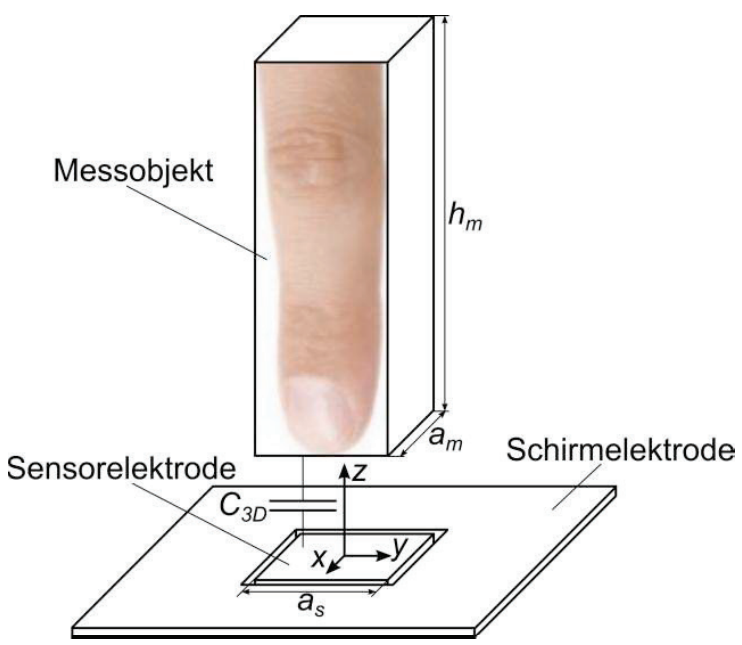

Abb. 1: Schematische Darstellung der Messanordnung.

In Abschnitt Modellierung wird zunächst das präsentierte 3D Problem durch Betrachtung der Symmetrieeigenschaften auf ein 2D Problem überführt. Darüber hinaus wird diese Konfiguration für eine mathematische Beschreibung des Kapazitätsverlaufs in weitere Teilprobleme gegliedert. Schließlich werden die Teillösungen des 2D Problems zu einem Kapazitätsmodell für drei Raumdimensionen zusammengefasst. Abschnitt Messungen beinhaltet Details zum Messaufbau und es werden die Messwerte mit dem Kapazitätsmodell verglichen. Darauf aufbauend werden schließlich in Abschnitt Erkenntnisse Schlussfolgerungen gezogen. Zuletzt werden in Abschnitt Ausblick weiterführende Problemstellungen erörtert.

\section{Modellierung}

Da eine analytische Berechnung von $C_{3 D}$ in Abb. 1 sehr aufwändig ist, werden durch Vereinfachungen Ersatzprobleme gelöst, die schließlich zu einer hinreichend genauen Näherung für $C_{3 D}$ führen. Dazu wird die Anordnung in Abb. 1 als ebenes Problem in der $x z^{-}$ Ebene für $y=0$ abstrahiert. Es wird ange-

\footnotetext{
${ }^{1}$ Die Plattenkondensatornäherung liefert für $1 \mathrm{~mm}$ Plattenabstand $2 \mathrm{pF}$.
}

nommen, dass in jeder zur $y$-Achse parallelen Ebene das gleiche Feld wirkt. Nun reicht es aus das 2D Problem zu lösen und zum Vergleich mit der originalen 3D Anordnung in Abb. 1 die 2D Größen mit der Ausdehnung $a_{y}$ in $y$-Richtung zu skalieren. Dieser Sachverhalt ist in Abb. 2 mit der Teilkapazität $C_{g}$ zwischen der Quadergrundfläche und der Sensorelektrode, der Teilkapazität $C_{d}$ zwischen der Quaderdeckelfläche und der Sensorelektrode sowie den Teilkapazitäten $C_{s l}$ bzw. $C_{s r}$ zwischen der linken bzw. rechten Quaderseitenfläche und der Sensorelektrode schematisch dargestellt. Mit $C_{2 D}$ wird die gesamte Kapazität zwischen Messobjekt und Sensorelektrode für das 2D Problem bezeichnet.

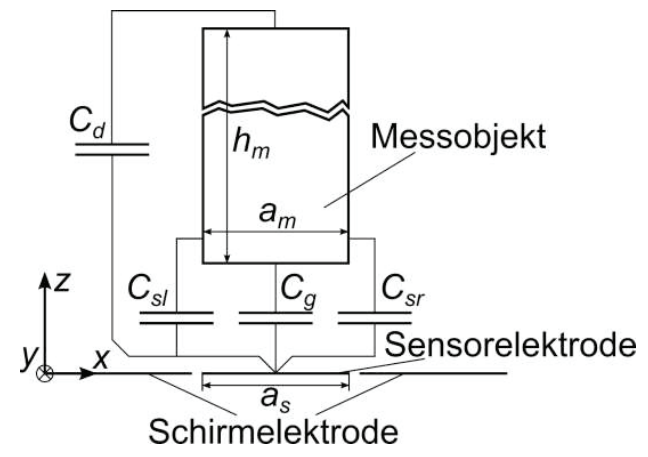

Abb. 2: Teilkapazitäten des 2D Problems.

Zur Verifikation wurden numerische Berechnungen mit dem Finite Elemente Methode (FEM) Programm COMSOL Multiphysics durchgeführt. Die Simulationen sollen unter anderem die Vereinfachungen für die Modellbildung plausibilisieren. Dabei wurden für die Abmessungen in Abb. 1 bzw. Abb. 2 die Werte in Tab. 1 verwendet. Für die 2D Simulationen wurde weiters $a_{y}=a_{s}$ benutzt. In der Simulation wird das Messobjekt über der Sensorelektrode in der $x z$-Ebene in einem Raster von $60 \mathrm{~mm}$ bis $20 \mathrm{~mm}$ äuqidistant mit $2 \mathrm{~mm}$ verschoben. In jedem dieser Punkte wird ein Kapazitätswert berechnet.

Tab. 1: Parameter für die Simulation.

\begin{tabular}{lcc}
\hline Beschreibung & Parameter & Wert \\
\hline Elektrodenbreite & $a_{s}$ & $15 \mathrm{~mm}$ \\
Quaderbreite & $a_{m}$ & $15 \mathrm{~mm}$ \\
Quaderhöhe & $h_{m}$ & $55 \mathrm{~mm}$ \\
\hline
\end{tabular}

Für die Modellierung werden folgende Vereinfachungen vorausgesetzt:

- Die Ausdehnung der Sensor- und Schirmelektroden in $z$-Richtung wird vernachlässigt.

- Der Spalt in der $x y$-Ebene zwischen Sensorelektrode und Schirmelektrode wird als infinitesimal modelliert. 
- Die Schirmelektrode erstreckt sich in der $x y$-Ebene bis ins Unendliche.

\subsection{Voruntersuchungen}

Abb. 3 zeigt die Simulation zum Betrag der relativen Abweichung von $C_{2 D}$ bezüglich $C_{3 D}$ für $y=0$. Da vor allem im Fernbereich große relative Abweichungen auftreten wurde zum Kapazitätsverlauf der 2D Simulation eine Konstante ergänzt. Diese berechnet sich durch Minimierung des Quadrats der relativen Abweichung. Damit erkennt man in Abb. 3, dass der Betrag der relativen Abweichung maximal $6 \%$ im betrachten Bereich annimmt. Grundsätzlich tritt im 3D Nahbereich ein höherer Kapazitätswert auf als im 2D Fall, weil hier noch zusätzliche Kapazitäten der Seitenflächen hinzukommen. Für den 3D Fernbereich nimmt die Kapazität hingegen stärker ab als für die 2D Anordnung. Wie bereits in der Einleitung erwähnt, spielt die tatsächliche Genauigkeit nur eine untergeordnete Rolle, so lange aus den Positionsdaten eine Tendenz zu einer bestimmten Geste erkennbar ist.

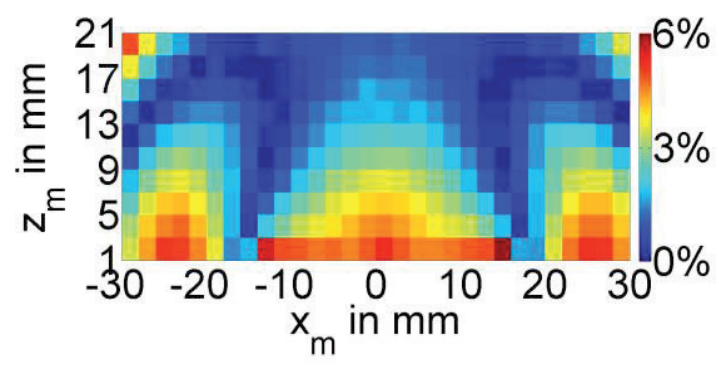

Abb. 3: Simulation zum Betrag der relativen Abweichung von $C_{2 D}$ bezüglich $C_{3 D}$ für $y=0$.

Die Kapazität zwischen Messobjekt und Sensorelektrode in Abb. 2 wird hauptsächlich durch die Position von drei Seiten des Metallquaders bestimmt, da für $h_{m} \gg$ die Deckelfläche kaum einen Einfluss hat. Abb. 4 zeigt die Simulation zum Betrag der relativen Abweichung von $C_{g}+C_{s l}+C_{s r}$ bezüglich $C_{2 D}$. Dabei wurden die aufsummierten Teilkapazitäten als Linienelemente simuliert. Wie bereits für Abb. 3 wurde auch hier zur Anpassung eine Konstante ergänzt. Diese berechnet sich durch Minimierung des Quadrats der relativen Abweichung. Der Betrag des relativen Fehlers bleibt über den betrachten Bereich unter $8 \%$, was für die Anwendung zur Gestenerkennung einen komfortablen Wert darstellt.

Die Voruntersuchungen verifizieren somit die Modellierung eines ebenen Problems anhand der Kapazitäten $C_{g}, C_{s l}$ und $C_{s r}$ aus Abb. 2. Die Verwendung der Linienelemente bildet eine elegante Möglichkeit, den mathematischen
Aufwand für die Modellierung in Grenzen zu halten.

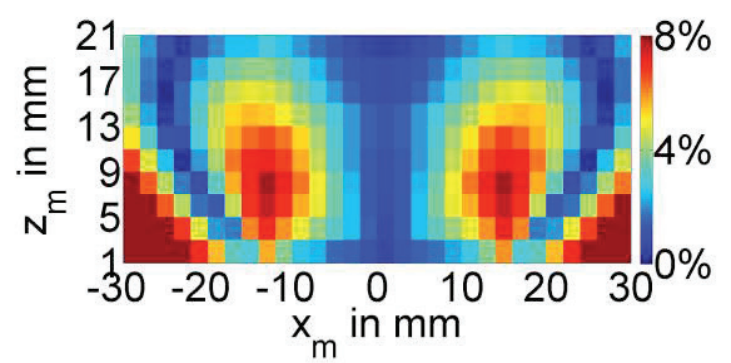

Abb. 4: Simulation zum Betrag der relativen Abweichung von $C_{g}+C_{s l}+C_{s r}$ bezüglich $C_{2 D}$.

\subsection{Bestimmung von $\boldsymbol{C}_{g}$}

Die Geometrie in Abb. 5 dient als Basis zur Berechnung der Kapazität $C_{g}$ zwischen Quadergrundfläche und Sensorelektrode aus Abb. 2. Es wird sich zeigen, dass durch Superposition zweier in $x$-Richtung verschobener Anordnungen $C_{g}$ berechnet werden kann. Die untere Elektrode 2 und der untere Schirm 2 repräsentieren dabei einen Teil der Sensorelektrode und der Schirmelektrode aus Abb. 2. Die obere Elektrode 1 stellt einen Teil der Quadergrundfläche aus Abb. 2 dar. Dort ist für den oberen Schirm 1 kein Pendant zu finden, da dieser in Abb. 5 zur virtuellen Abschirmung der linken seitlichen Teilkapazitäten $C_{s l}$ bzw. im verschobenen Fall zur virtuellen Abschirmung von $C_{s r}$ und zur einfacheren mathematischen Berechnung (Superposition) dient. Der Schirm 1 trifft bei $z=z_{d}$ und $x=x_{1}$ auf die Elektrode 1 (rot). Der Schirm 2 trifft bei $z=0$ und $x=x_{2}$ auf die Elektrode 2 (rot). Zwischen den beiden Elektroden 1 und 2 liegt die Spannung $U_{12}$. Weiters ist für die Berechnung der Potentialverteilung die Anordnung in den Bereich I $\left(x<x_{1}\right)$ und den Bereich II $\left(x>x_{1}\right)$ aufgeteilt.

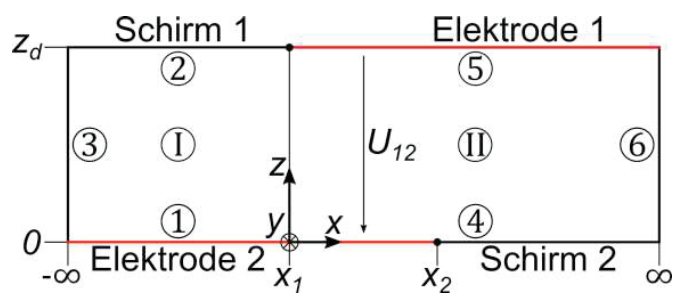

\section{Abb. 5: Geometrie als Basis zur Berechnung} der Kapazität $C_{g}$.

Das Potential $\varphi$ innerhalb der Elektrodenanordnung in Abb. 5 ergibt sich als

$$
\varphi(x, z)= \begin{cases}\varphi_{\mathrm{I}}(x, z) & \text { für } x \leq x_{1} \\ \varphi_{\mathrm{II}}(x, z) & \text { für } x>x_{1}\end{cases}
$$


mit dem Potential $\varphi_{\mathrm{I}}$ im Bereich I und dem Potential $\varphi_{\text {II }}$ im Bereich II. Die Ränder werden auf die Bereiche (1)bis (6)aufgeteilt mit den Potentialen laut Tab. 2. Zur eindeutigen Lösung der Laplacegleichung [7] der Form

$$
\Delta \varphi(\mathrm{x}, \mathrm{z})=0
$$

sind zusätzlich zu den Randbedingungen in Tab. 2 noch Forderungen an den Grenzbereich zwischen I und II notwendig. Das ist zum einen die Dirichletbedingung

$$
\varphi_{\mathrm{I}}\left(x_{1}, z\right)=\varphi_{\mathrm{II}}\left(x_{1}, z\right) \text { für } 0<z<z_{d}
$$

und zum anderen die Neumannbedingung

$\frac{d \varphi_{\mathrm{I}}\left(x_{1}, z\right)}{d x}=\frac{d \varphi_{\mathrm{II}}}{d x}\left(x_{1}, z\right)$ für $0<z<z_{d}$.

Tab. 2: Potentiale der Ränder (1) bis (6).

\begin{tabular}{cccc}
\hline Rand & $x$ & $z$ & $\varphi$ \\
\hline (1) & $x<x_{1}$ & $z=0$ & 0 \\
(2) & $x<x_{1}$ & $z=z_{d}$ & 0 \\
(3) & $x \rightarrow-\infty$ & $0<z<z_{d}$ & 0 \\
(4) & $x>x_{1}$ & $z=0$ & 0 \\
(5) & $x>x_{1}$ & $z=z_{d}$ & $U_{12}$ \\
(6) & $x \rightarrow \infty$ & $0<z<z_{d}$ & $U_{12} \frac{z}{z_{d}}$ \\
\hline
\end{tabular}

In [8] wird für die Lösung von (2) mit den Randbedingungen in Tab. 2 sowie den Grenzbedingungen in (3) und (4) ein Produktansatz vorgeschlagen. Dies führt schließlich zu den Teilpotentialen $\varphi_{\mathrm{I}}$ und $\varphi_{\mathrm{II}}$ der Gestalt

$$
\begin{gathered}
\varphi_{\mathrm{I}}(x, z)= \\
\frac{U_{12}}{\pi} \sum_{n=1}^{\infty} \frac{(-1)^{n+1}}{n} e^{\frac{-n \pi\left|x-x_{1}\right|}{z_{d}}} \sin \left(\frac{n \pi z}{z_{d}}\right) \\
\varphi_{\mathrm{II}}(x, z)=U_{12} \frac{z}{z_{d}}-\varphi_{\mathrm{I}}(x, z) .
\end{gathered}
$$

Im Weiteren wird mit (5) die Flächenladungsdichte $\sigma_{2}$ auf der Elektrode 2 in Abb. 5 zu

$$
\sigma_{2}(x)=-\varepsilon_{0} \frac{\partial \varphi(x, 0)}{\partial z}=-\varepsilon_{0} \frac{U_{12}}{z_{d}} \frac{e^{\frac{\pi\left(x-x_{1}\right)}{z_{d}}}}{1+e^{\frac{\pi\left(x-x_{1}\right)}{z_{d}}}}
$$

berechnet. Durch Superposition einer Konfiguration nach $\mathrm{Abb} .5$ mit

$$
x_{1}=x_{m}-\frac{a_{m}}{2}, \quad x_{2}=\frac{a_{s}}{2}
$$

bei geänderter positiver Zählpfeilrichtung von $U_{12}$ und einer mit

$$
x_{1}=x_{m}+\frac{a_{m}}{2}, \quad x_{2}=-\frac{a_{s}}{2}
$$

resultiert die Geometrie zur Berechnung von $C_{g}$ aus Abb. 2. Die Flächenladungsdichte $\sigma_{\mathrm{s}}$ der Sensorelektrode lässt sich dann durch Superposition der Konfigurationen über

$$
\sigma_{s}(x)=-\left.\sigma_{2}(x)\right|_{x_{1}=x_{m}-\frac{a_{m}}{2}}+\left.\sigma_{2}(x)\right|_{x_{1}=x_{m}+\frac{a_{m}}{2}}(9)
$$

angeben. Mithilfe von (9) kann die elektrische Ladung $Q_{\mathrm{s}}$ auf der Sensorelektrode als

$$
Q_{s}=a_{y} \int_{-\frac{a_{s}}{2}}^{\frac{a_{s}}{2}} \sigma_{s}(x) \mathrm{d} x
$$

berechnet werden. Damit lässt sich $C_{g}$ über die Kapazitätsdefinition

$$
C_{g}=\frac{Q_{s}}{U_{12}}
$$

$\mathrm{zu}$

$$
C_{g}=a_{y} \frac{\varepsilon_{0}}{\pi} \ln \left(\frac{\cosh \left(k_{g 1}\right) \cosh \left(k_{g 2}\right)}{\cosh \left(k_{g 3}\right) \cosh \left(k_{4 g}\right)}\right)
$$

bestimmen. Die Parameter $k_{g 1}, k_{g 2}, k_{g 3}$ und $k_{g 4}$ sind dabei durch

$$
\begin{aligned}
& k_{g 1}=\frac{\pi}{4 z_{d}}\left(2 x_{m}-a_{s}-a_{m}\right) \\
& k_{g 2}=\frac{\pi}{4 z_{d}}\left(2 x_{m}+a_{s}+a_{m}\right) \\
& k_{g 3}=\frac{\pi}{4 z_{d}}\left(2 x_{m}+a_{s}-a_{m}\right) \\
& k_{g 4}=\frac{\pi}{4 z_{d}}\left(2 x_{m}-a_{s}+a_{m}\right)
\end{aligned}
$$

festgelegt. Für gleiche Abmessungen des Messobjekts und der Sensorelektrode $a_{m}=a_{s}$ kann man $C_{g}$ an der Stelle $x_{m}=0 \mathrm{zu}$

$$
C_{g}=a_{y} \frac{\varepsilon_{0}}{\pi} \ln \left(\frac{1}{2} \cosh \left(\frac{\pi a_{s}}{z_{d}}\right)+\frac{1}{2}\right)
$$

vereinfachen. Wird des Weiteren noch $a_{s} / z_{d} \gg 1$ vorausgesetzt, ergibt sich für $C_{g}$ näherungsweise der bekannte Ausdruck für die Kapazität eines Plattenkondensators

$$
C_{g} \approx \varepsilon_{0} \frac{a_{y} a_{s}}{z_{d}}
$$

\subsection{Bestimmung von $C_{s l}$ und $C_{s r}$}

Die konforme Abbildung $f$ aus $[9,10]$, der Form

$$
t=f(s)=\ln (s)
$$

in Abb. 6 dient als Grundlage zur Berechnung der Kapazitäten $C_{s l}$ und $C_{s r}$. Dabei erfolgt eine Transformation von der $s$-Ebene

$$
s=x+j z
$$

in die $t$-Ebene

$$
t=\xi+j \varsigma .
$$


Durch diese Transformation wird in Abb. 6 die radiale Geometrie mit den Radien $r_{o 1}, r_{o 2}, r_{u 1}$ und $r_{u 2}$ sowie dem Winkel $\alpha$ in der $s$-Ebene auf ein planares Problem in der $t$-Ebene abgebildet. Somit liegt in der $t$-Ebene ein äquivalentes Problem wie bei der Berechnung von $C_{g}$ vor.

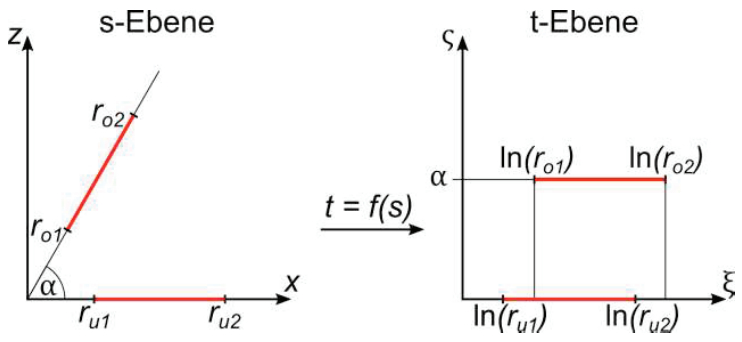

Abb. 6: Konforme Abbildung.

Für die Anwendung der Abbildungsvorschrift aus (16) zur Berechnung von $C_{s r}$ werden drei von der Messobjektposition abhängige Bereiche (vgl. Abb. 1 und Abb. 2) der Gestalt

Bereich 1: $-\frac{a_{s}+a_{m}}{2}>x_{m}$

Bereich 2: $-\frac{a_{s}+a_{m}}{2} \leq x_{m}<\frac{a_{s}-a_{m}}{2}$

Bereich 3: $\frac{a_{s}-a_{m}}{2} \leq x_{m}$

unterschieden. In Abb. 7 sind schematische Darstellungen für die Bereiche 1 und 2 abgebildet. Es wird ein relatives Koordinatensystem mit den Achsen $x^{\prime}$ und $z^{\prime}$ eingeführt. Dieses bewegt sich in $x$-Richtung mit dem Messobjekt mit.

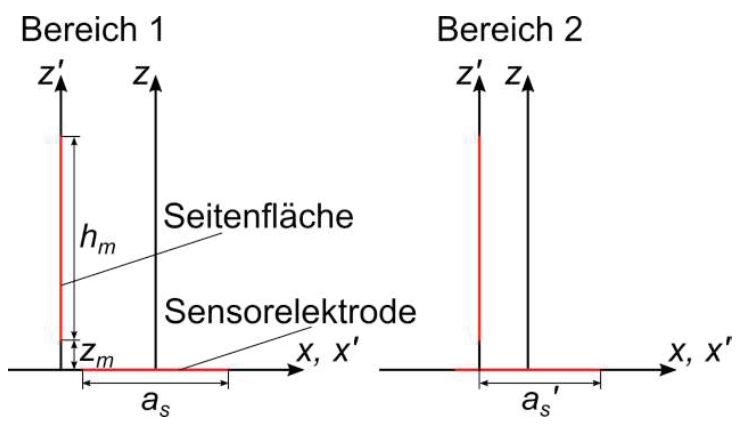

Abb. 7: Schematische Darstellung für die Modellierung der Kapazität $C_{s r}$.

Damit ergeben sich für den Bereich 1 die Parameter in Abb. 6 zu

$$
\begin{gathered}
r_{o 1}=z_{m}, \quad r_{o 2}=r_{o 1}+h_{m} \\
r_{u 1}=-\frac{a_{s}+a_{m}}{2}-x_{m}, r_{u 2}=r_{u 1}+a_{s} \\
\alpha=\frac{\pi}{2} .
\end{gathered}
$$

Im Bereich 2 verkleinert sich die effektive Sensorelektrodenbreite von $a_{s}$ auf $a_{s}{ }^{\prime}$. Die Parameter in Abb. 6 folgen dann als

$$
r_{u 1}=0, \quad r_{u 2}=a_{s}{ }^{\prime},
$$

wobei (20.a) und (20.c) unverändert bleiben. Bereich 3 ist in Abb. 7 nicht abgebildet, da $a_{s}{ }^{\prime}=0$ gilt. Hiermit ergeben sich die Parameter in Abb. 6 zu

$$
r_{u 1}=0, \quad r_{u 2}=0,
$$

womit keine Kapazitätsbeiträge mehr auftreten. So liegt mit (20) bzw. (21) in der $t$-Ebene (vgl. Abb. 6) für die Kapazitätsberechnung von $C_{s r}$ dasselbe Problem vor wie für $C_{g}$. Analog zu (12) und (13) ergibt sich damit für $C_{s r}$ der Ausdruck

$$
C_{s r}=a_{y} \frac{\varepsilon}{\pi} \ln \left(\frac{\cosh \left(k_{s r 1}\right) \cosh \left(k_{s r 2}\right)}{\cosh \left(k_{s r 3}\right) \cosh \left(k_{s r 4}\right)}\right)
$$

wobei mit (20) und (21) bzw. (22) die Parameter $k_{s r 1}, k_{s r 2}, k_{s r 3}$ und $k_{s r 4}$ in der Form

$$
\begin{aligned}
& k_{\mathrm{sr} 1}=\frac{\pi}{2 \alpha}\left(\ln \left(r_{u 2}\right)-\ln \left(r_{o 1}\right)\right) \\
& k_{\mathrm{sr} 2}=\frac{\pi}{2 \alpha}\left(\ln \left(r_{u 1}\right)-\ln \left(r_{o 2}\right)\right) \\
& k_{\mathrm{sr} 3}=\frac{\pi}{2 \alpha}\left(\ln \left(r_{u 1}\right)-\ln \left(r_{o 1}\right)\right) \\
& k_{\mathrm{sr} 4}=\frac{\pi}{2 \alpha}\left(\ln \left(r_{u 2}\right)-\ln \left(r_{o 2}\right)\right)
\end{aligned}
$$

folgen. Für den Bereich 2 ist zu ergänzen, dass aufgrund der Verkleinerung der effektiven Elektrodenbreite von $a_{s}$ auf $a_{s}{ }^{\prime}$ Kapazitätsanteile vernachlässigt werden. Außerdem wird im Bereich 3 zur Gänze von Kapazitätsbeiträgen abgesehen.

Die Kapazitätsberechnung von $C_{s l}$ wird analog zu $C_{s r}$ in (23) durchgeführt.

\subsection{Gesamtmodell}

Zunächst wird mit (12) und (13) sowie mit (23) und (24) das 2D Kapazitätsmodell $C_{2 D}$ in der Form

$$
C_{2 D}\left(x_{m}, z_{m}\right)=C_{g}+C_{s l}+C_{s r}
$$

hergeleitet. Abb. 8 zeigt eine Gegenüberstellung von Simulation und Modell zu $C_{2 D}$. Wie bereits erwähnt, werden für die Kapazitäten zwischen Quaderseitenfläche und Sensorelektrode in (19) drei Bereiche unterschieden. Aufgrund der getroffenen Annahmen bei der Modellierung werden für $C_{s l}$ in den Bereichen 1 und $2 \mathrm{bzw}$. für $C_{s r}$ in den Bereichen 2 und 3 Kapazitätsanteile vernachlässigt. Für den Nahbereich wirkt sich die relative Kapazitäts-

\footnotetext{
${ }^{2}$ Das ist der triviale Fall $C_{s r}=0$.
} 
abweichung aufgrund des hohen Wertes von $C_{g}$ kaum aus. Im Fernbereich sind die Kapazitätsanteile $C_{g}$ bzw. $C_{s l}$ und $C_{s r}$ in der gleichen Größenordnung, d.h. obwohl die absolute Abweichung sinkt, steigt die relative Abweichung.

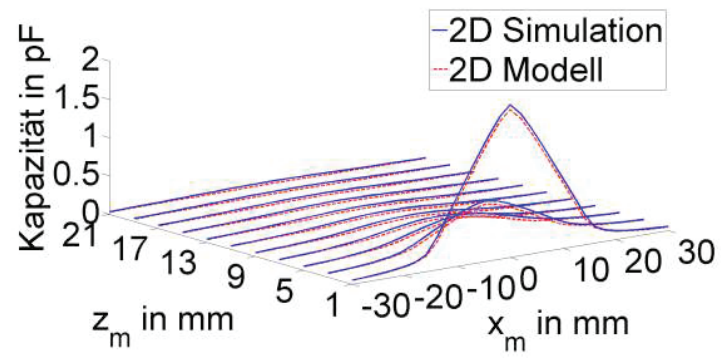

Abb. 8: Gegenüberstellung von Simulation und Modell zu $C_{2 D}$.

Nun stellt sich die Frage nach der Erweiterung des 2D Kapazitätsmodells auf drei Raumdimensionen. Dafür werden die KapazitätsIsolinien $^{3}$ der Simulation zu $C_{3 D}$ für $z_{m}=2 \mathrm{~mm}$ und $z_{m}=5 \mathrm{~mm}$ in Abb. 9 betrachtet. Man erkennt, dass die Isolinien mit zunehmendem Abstand kreisförmig werden. Im Nahbereich folgt als Konsequenz der quadratischen Form von Sensorelektrode und Quadergrundfläche sowie durch die zusätzlichen Quaderseitenflächen eine Abweichung von der Kreisform.

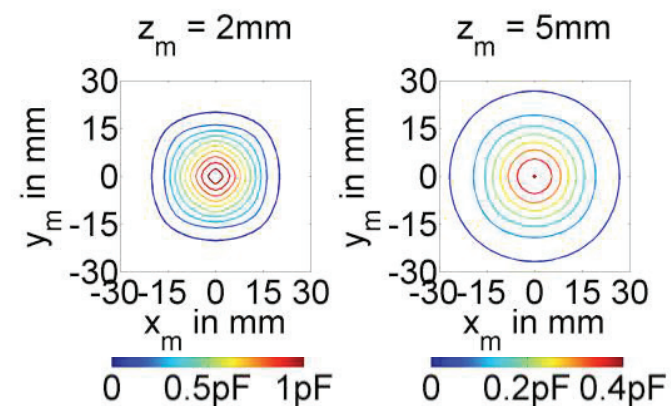

Abb. 9: Kapazitäts-Isolinien der Simulation zu $C_{3 D}$ für $z_{m}=2 \mathrm{~mm}$ und $z_{m}=5 \mathrm{~mm}$.

In Abb. 9 sind für $z_{m}=2 \mathrm{~mm}$ noch Abweichungen von der Kreisform zu erkennen. Jedoch ergeben sich für $z_{m}=5 \mathrm{~mm}$ bereits näherungsweise kreisförmige Kapazitäts-Isolinien in der $x y$-Ebene. Daher wird das 3D Kapazitätsmodell $C_{3 D}$ mit (25) in der Gestalt

$$
C_{3 D}\left(x_{m}, y_{m}, z_{m}\right)=C_{2 D}\left(\sqrt{x_{m}^{2}+y_{m}^{2}}, z_{m}\right)
$$

angegeben (vgl. [11]). Die Qualität des Kapazitätsmodells in (26) wird im Weiteren auch mithilfe von Messungen überprüft.

\footnotetext{
${ }^{3}$ Das sind Linien, auf denen jeweils an jedem Punkt der gleiche Kapazitätswert auftritt.
}

\section{Kapazitätsmessung}

Die Kapazitätsmessungen werden mit dem Evaluierungsboard des Kapazitäts-DigitalUmsetzers AD7747 durchgeführt. Dieser hat eine Messunsicherheit von $\pm 4 \mathrm{fF}$. Die Auswertung der Messdaten erfolgt in Matlab. Der verwendete Messaufbau wurde bereits in Abb. 1 schematisch dargestellt. Für die Reproduzierbarkeit der Messungen wird das Messobjekt mit einem 3-Achssystem über der Sensorelektrode präzise positioniert. Die Auflösung dieses Positioniersystems ist dabei im Submillimeterbereich. Abb. 10 zeigt das auf einem Ausleger montierte Messobjekt (links) und die verwendete Sensorelektrode mit Schirm (rechts).
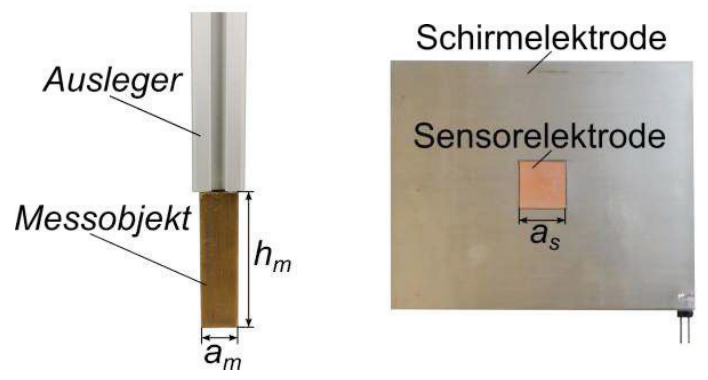

Abb. 10: Auf Ausleger montiertes Messobjekt (links) und Sensorelektrode mit Schirm (rechts).

Das Messobjekt ist dabei, wie bereits erwähnt, geerdet und soll einen Finger abstrahieren. Die Senorelektrode wurde aus einer doppeltseitig beschichteten Leiterplatine gefertigt. Die Unterseite dient zur Schirmung. Auf der Oberseite befindet sich die Sensorelektorde mit der Zuleitung. Zur Unterdrückung der Zuleitungseinflüsse wurde auf der Oberseite zusätzlich eine mit Nanosilber bedruckte PET-Folie zur Schirmung montiert. Die Sensorelektrode wird mit dem Messsignal des AD7747 beaufschlagt. Die Schirmflächen werden über einen Verstärker auf gleichem Potential wie die Sensorelektrode gehalten. Der konstante Kapazitätswert $C_{o}$ für ein weit entferntes Messobjekt ${ }^{4}$ wird als Offset bezeichnet. Dieser wird bei Abwesenheit des Messobjekts bestimmt und bei jedem Messpunkt von den tatsächlich gemessenen Kapazitätswerten abgezogen. Die hier verwendete Annahme der Unabhängigkeit von $C_{o}$ und der Messposition ist eine oft benutzte Näherung [12]. Für die Aufnahme der Messreihen wurde ein Messvolumen von $32 \mathrm{~mm}$ x $32 \mathrm{~mm}$ x $20 \mathrm{~mm}$ mit äquidistanten Abständen der Messpunkte von $2 \mathrm{~mm}$ verwendet. Diese Abmessungen wurden in der $x y$-Ebene so gewählt, dass sich beim schachbrettartigen Anordnen von Sensorelektroden die Messvolumen auf einem

\footnotetext{
${ }^{4}$ Der Messwert ändert sich für größere Entfernung nur noch im Bereich des Rauschens.
} 


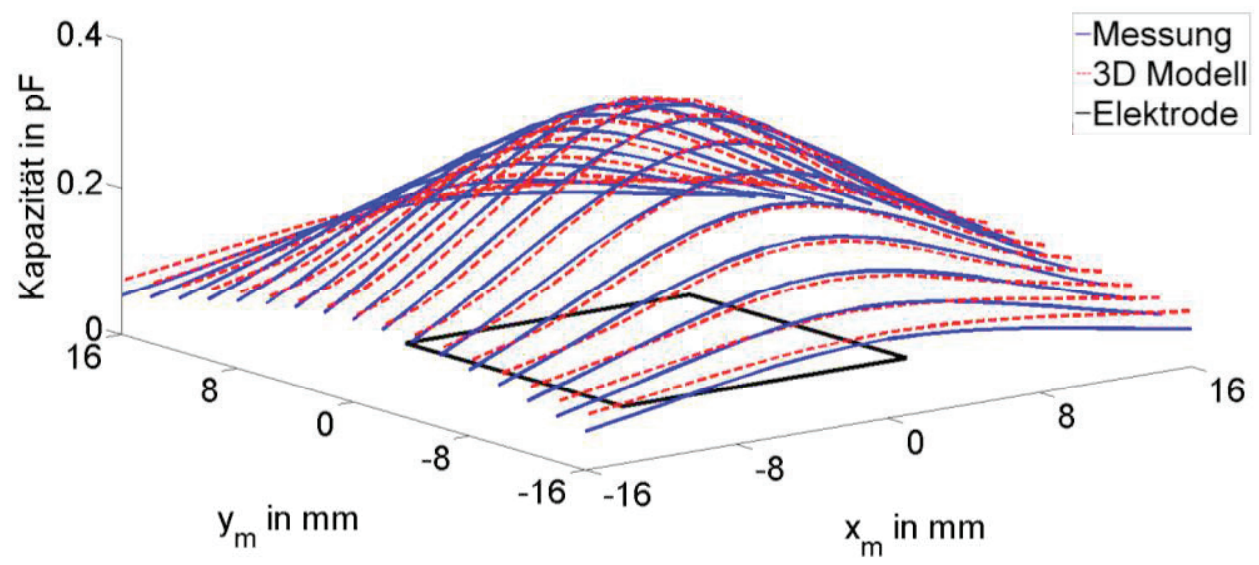

Abb. 11: Gegenüberstellung von Messung zu Modell für $C_{3 D}$ bei $z_{m}=5 \mathrm{~mm}$.

großen Bereich überschneiden. Dies ist für nachfolgende Arbeiten von Interesse, bei denen man z. B. mithilfe von Mehrelektrodengeometrien auf die Position von Messobjekten schließen will. Hier ist eine hohe Anzahl an Messwerten in einem definierten Bereich günstig.

Eine Gegenüberstellung von Messung zu Modell für $C_{3 D}$ bei $z_{m}=5 \mathrm{~mm}$ ist in Abb. $11 \mathrm{zu}$ sehen. Im betrachteten Bereich erkennt man dabei eine gute Übereinstimmung von Messung und Modell. Die Abweichungen resultieren zum einen aus vernachlässigten Geometrieparameter für die Modellherleitung (vgl. Abb. 1 und Abb. 10) und zum anderen aus den in Abschnitt Modellierung plausibilisierten Vereinfachungen. Darüber hinaus liefert die Näherung eines konstanten Wertes $C_{o}$ ebenfalls einen Beitrag der nicht berücksichtigt wurde.

In Abb. 12 ist der Betrag der relativen Abweichung des Modells bezüglich der Messung für $C_{3 D}$ bei $z_{m}=5 \mathrm{~mm}$ und $z_{m}=15 \mathrm{~mm}$ dargestellt.

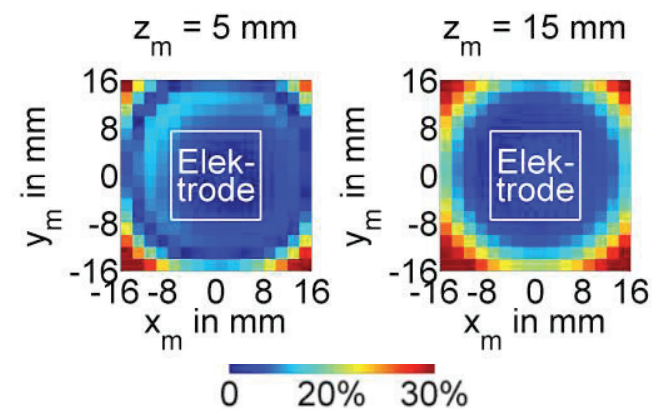

Abb. 12: Betrag der relativen Abweichung des Modells bezüglich der Messung für $C_{3 D}$ bei $z_{m}=5 \mathrm{~mm}$ und $z_{m}=15 \mathrm{~mm}$.

Der Betrag der maximalen relativen Abweichung bleibt dabei über den gesamten Bereich unter $30 \%$ bzw. im Bereich um die Sensorelektrode unter $10 \%$. Die absolute Abweichung nimmt mit dem Abstand zum Koordinatenursprung auf der Sensorelektrode zwar ab, jedoch sinkt der Messwert stärker, womit der relative Fehler zunimmt. Die auftretenden Abweichungen sind für eine Positionsbestimmung zur Gestenerkennung tolerierbar, da die Kenntnis der exakten Absolutposition nicht notwendig ist. Einer definierten zeitlichen $A b$ folge von relativen Positionen wird eine Geste zugeordnet. Hier ist vor allem eine geringe Rechenzeit für die Auswertung schneller Änderungen der Fingerposition ein entscheidendes Kriterium. Dabei bietet (26) eine gute Möglichkeit, mit zwar beschränkter Genauigkeit aber dafür rasch Positionen zu bestimmen.

$\mathrm{Zu}$ Abb. 12 sei angemerkt, dass es sich um Messwerte handelt und auch das Modell in (26) nicht an die Messung angepasst wurde. Dies lässt noch Verbesserungsmöglichkeiten durch empirische Adaptierungsparameter offen.

\section{Erkenntnisse}

Die wesentlichen Erkenntnisse dieser Arbeit sind:

- Die Berechnung der Kapazität zwischen Messobjekt und Sensorelektrode in Abb. 1 kann in der $x z$-Ebene für $y_{m}=0$ näherungsweise mithilfe der ebenen $2 \mathrm{D}$ Anordnung in Abb. 2 erfolgen.

- Der Kapazitätsverlauf der 2D Konfiguration in Abb. 2 kann durch die Teilkapazitäten $C_{g}, C_{s l}$ und $C_{s r}$ approximiert werden.

- Unter Annahme kreisförmiger KapazitätsIsolinien (siehe Abb. 9) ist das 2D Kapazitätsmodell in (25) auf 3 Raumdimensionen zu (26) erweiterbar.

- Für die angenommene Geometrie in Abb. 1 eignet sich das Modell (26) für weitere Untersuchungen zur Positionsbestimmung für die Gestenerkennung. 


\section{Ausblick}

In weiterführenden Arbeiten sollen hinsichtlich der Modellierung

- die Abhängigkeit des Kapazitätsoffsets von der Messobjektposition,

- Verdrehungen des Messobjekts um die $x$-, $y$ - und $z$-Achse,

- Metallquader mit beliebigem Seitenverhältnis der Grundfläche und

- eine bessere Approximation der Kapazitäts-Isolinien

untersucht werden. Weiters ist eine Erweiterung des Elektrodendesigns in Abb. 10 auf Mehrelektrodenstrukturen notwendig. Anschließend soll mit Parameterschätzverfahren oder Machine Learning Ansätzen eine Positionsbestimmung und darauf aufbauend eine Erkennung menschlicher Gesten realisiert werden.

Danksagung: Diese Arbeit wurde größtenteils durch die Österreichische Forschungsförderungsgesellschaft $\mathrm{mbH}$ (FFG) mit der Projektnummer 846057 und durch die Firma WimTec Sanitärprodukte $\mathrm{GmbH}$ finanziert. Außerdem möchte ich mich bei Herrn Simon Hehenberger für die Aufnahme der Messwerte bedanken.

\section{Literatur}

[1] A. Braun, T. Dutz, F. Kamieth, Capacitive sensor-based hand gesture recognition in ambient intelligence scenarios, PETRA '13 Proceedings of the 6th International Conference on PErvasive Technologies Related to Assistive Environments; doi: 10.1145/2504335.2504340

[2] T. Grosse-Puppendahl, A. Marinc, A. Braun, Classification of User Postures with Capacitive Proximity Sensors in AAL-Environments, Ambient Intelligence, Lecture Notes in Computer Science, vol. 7040, 314-323, Springer (2011)

[3] T. Große-Puppendahl, A. Braun, Honeyfish - A High Resolution Gesture Recognition System based on Capacitive Proximity Sensing, EmbeddedWorld Conference, Weka Fachmedien (2012)

[4] A. K. Jain, Fundamentals of Digital Image Processing, John Wiley \& Sons (2010); ISBN10: 0470844736

[5] T. Große-Puppendahl et. al, Ambient GestureRecognizing Surfaces with Visual Feedback, Distributed, Ambient and Pervasive Interactions 2014, Lecture Notes in Computer Science, Springer (2014)

[6] G. Durcansky, EMV-gerechtes Gerätedesign, Franzis Verlag GmbH (1999); ISBN-10: 3772353886

[7] J. D. Jackson, Klassische Elektrodynamik, Walter de Gruyter GmbH \& Co. KG (2006); ISBN-10: 3110189704

[8] G. Willem de Jong, Smart Capacitive Sensors, Dissertation (1994), Technische Universität Delft
[9] P. Henrici, Applied and Computational Complex Analysis, John Wiley \& Son (1974); ISBN: 978-0-471-54289-6

[10] N. Eidenberger, Konforme Abbildungen zur Lösung ebener inverser Probleme in der Messtechnik, Dissertation (2014), Johannes Kepler Universität Linz

[11] T. Grosse-Puppendahl, Capacitive Sensing and Communication for Ubiquitous Interaction and Environmental Perception, Dissertation (2013), TU Darmstadt

[12] T. Grosse-Puppendahl et. al, OpenCapSense: A Rapid Prototyping Toolkit for Pervasive Interaction using Capacitive Sensing, IEEE International Conference on Pervasive Computing and Communications, IEEE (2013); doi: 10.1109/PerCom.2013.6526726 